\title{
Ebselen protects rat hearts against myocardial ischemia-reperfusion injury
}

\author{
BIN CHENG $^{1^{*}}$, JIN-PENG ZHONG ${ }^{2 *}$, FU-XIA WU ${ }^{2}$, GUAN-LAN LI ${ }^{2}$, \\ QING-XIAO RUAN ${ }^{2}$, GANG LUO ${ }^{2}$ and HONG JIANG ${ }^{1}$ \\ ${ }^{1}$ Department of Cardiology, Renmin Hospital of Wuhan University, Wuhan, Hubei 430060; ${ }^{2}$ Department of Cardiology,
The People's Hospital of China Three Gorges University, Yichang, Hubei 443000, P.R. China
}

Received May 28, 2018; Accepted November 9, 2018

DOI: $10.3892 /$ etm.2018.7089

\begin{abstract}
Ebselen is an organoselenium compound that has demonstrated potent antioxidant and anti-inflammatory effects in previous studies. The present study was conducted to evaluate the effect of ebselen on myocardial ischemia-reperfusion $(\mathrm{I} / \mathrm{R})$ injury in a rat model and to elucidate the related mechanisms. Myocardial infarct size was assessed using triphenyltetrazolium chloride staining. Myocardial injury was evaluated according to the histopathological and ultrastructural alterations of rat hearts and the serum activity levels of cardiac enzymes, including creatine kinase (CK), CK-MB isoenzyme and lactate dehydrogenase (LDH). Cardiomyocyte apoptosis was detected using the terminal dUTP nick end-labelling (TUNEL) assay. In addition, the expression of apoptosis-associated proteins was measured using western blot analysis. In heart tissue specimens the activity of superoxide dismutase (SOD) and glutathione peroxidase (GPx), and levels of malondialdehyde (MDA) and protein carbonyl (PC) were also detected. The results indicated that ebselen reduced I/R-induced increase in myocardial infarct size and prevented
\end{abstract}

Correspondence to: Professor Hong Jiang, Department of Cardiology, Renmin Hospital of Wuhan University, 238 Jiefang Road, Wuhan, Hubei 430060, P.R. China

E-mail: hong-jiang@whu.edu.cn

*Contributed equally

Abbreviations: I/R, ischemia reperfusion; TUNEL, terminal dUTP nick end-labelling; TTC, triphenyltetrazolium chloride; SOD, superoxide dismutase; GPx, glutathione peroxidase; MDA, malondialdehyde; PC, protein carbonyl; ROS, reactive oxygen species; RNS, reactive nitrogen species; CK[-MB], creatine kinase [MB isoenzyme]; LDH, lactate dehydrogenase; Bcl-2, B-cell lymphoma 2; Bax, B-cell lymphoma 2-associated X protein; PARP, poly(ADP) ribose; $\mathrm{C}$-, cleaved; $\mathrm{EF} \%$, ejection fraction; \%FS, percent fractional shortening; IA, infarct area; AAR, area at risk; PBS, phosphate-buffered saline

Key words: ebselen, myocardial, ischemia-reperfusion, apoptosis, antioxidant the I/R-induced decreases in ejection fraction and fractional shortening. Further of note, ebselen improved I/R-induced rat heart injury. This was indicated by attenuation of histological and ultrastructural changes; reduction of serum CK, CK-MB and LDH activity levels; and decreased cell apoptosis on TUNEL staining, which was verified by decreased expression of cleaved (C)-Caspase-8, C-Caspase-3, B-cell lymphoma 2 (Bcl-2)-associated X protein and C-PARP, and increased expression of Bcl-2. Additionally, SOD and GPx activity levels were significantly higher, while MDA and PC levels were significantly lower in the ebselen $+\mathrm{I} / \mathrm{R}$ group compared with in the I/R group. In conclusion, the present results suggested that ebselen serves an important role in protecting against myocardial I/R injury. The underlying mechanism may involve suppression of cardiomyocyte apoptosis and promotion of antioxidant activity.

\section{Introduction}

Interruption of blood supply to tissues results in ischemic injury. However, restoration of the blood supply initiates a chain of events that may result in additional cell injury, which is known as reperfusion injury $(1,2)$. Myocardial ischemia-reperfusion (I/R) injury has crucial roles in inducing cardiomyocyte apoptosis, necrosis and left ventricular remodeling, which can result in irreversible consequences, including myocardial infarction, progressive deterioration of cardiac function and heart failure $(3,4)$. In myocardial I/R injury, redox imbalances trigger a number of signaling pathways mediated by free radicals including reactive nitrogen species (RNS) and reactive oxygen species (ROS), such as superoxide anions, hydroxyl radicals, hydrogen peroxide, nitric oxide and peroxynitrite (5-7). In myocardial ischemia, hypoxia and reoxygenation, which may induce the excessive production of RNS and ROS in cardiac tissues, are principal causes of reperfusion injury (8). Free radicals are typically scavenged by antioxidant enzymes, including superoxide dismutase (SOD), catalase and glutathione peroxidase (GPx), which serve important roles in the prevention of lipid peroxidation $(9,10)$. Furthermore, previous studies have revealed that the antioxidant activities of these enzymes may be reduced in patients following myocardial infarction or in those with ischemic heart disease $(11,12)$. 
Previous studies have reported some effective strategies for protecting the heart against I/R injury in rat models; however, these strategies have been mostly unsatisfactory in limiting oxidative damage in clinical trials (13-15). Ebselen [2-phenyl-1, 2-benzisoselenazol-3(2H)-one] is a selenium-containing organic compound that is described as a GPX mimic and has protective effects against oxidative injury $(16,17)$. Since oxygen free radical scavengers serve an important role in the attenuation of I/R injury, the present study evaluated the effects of ebselen, a potent scavenger of peroxynitrite, against myocardial I/R injury in a rat model.

\section{Materials and methods}

Animals and myocardial $I / R$ model. A total of 32 male Sprague-Dawley rats (260-300 g, 3-4 weeks old) were obtained from the Laboratory Animal Center of Wuhan University, Wuhan, China. The rats were maintained with standard feeding conditions including adequate food and water ad libitum, a $12 \mathrm{~h} \mathrm{light/dark,} 20-25^{\circ} \mathrm{C}$ and 50-65\% humidity. Following a 12-h fasting period, all rats were anesthetized with $10 \%$ ketamine $(80 \mathrm{mg} / \mathrm{kg}$; Jiangsu Hengrui Pharmaceutical Co., Ltd., Lianyungang, China) and $2 \%$ xylazine $(5 \mathrm{mg} / \mathrm{kg}$; Sigma-Aldrich; Merck KGaA, Darmstadt, Germany) by intraperitoneal injection. Subsequently, surgery was performed on rats at room temperature $\left(22-24^{\circ} \mathrm{C}\right)$. Following skin incision, the hearts were exposed through a left thoracotomy in the fourth intercostal space. A 6-0 silk suture was placed $1-2 \mathrm{~cm}$ from the root of the left anterior descending coronary artery. The suture was loosened following occlusion for $30 \mathrm{~min}$, which was followed by $2 \mathrm{~h}$ of reperfusion. The same surgical procedures without ligation were performed on rats in a sham group. Following this, the skin was sutured, anesthesia was discontinued and the rats were put into pre-warmed cages for recovery. Rats were sacrificed by cervical dislocation under anesthesia with $5.0 \%$ isoflurane at the end of reperfusion $\sim 3 \mathrm{~h}$ after surgery. Blood samples and heart tissues were collected for further analysis.

All experiments were performed in accordance with protocols approved by the Laboratory Animal Ethics Committee of Wuhan University. All experimental animals received care in compliance with the Guide for the Care and Use of Laboratory Animals of the National Institute of Health (18).

Experimental procedures. Rats were equally and randomly divided into four groups as follows: A sham-operated group (sham group, $\mathrm{n}=8)$; an $\mathrm{I} / \mathrm{R}$ group $(\mathrm{n}=8)$; an ebselen $+\mathrm{I} / \mathrm{R}$ group (20 $\mathrm{mg} / \mathrm{kg}$ ebselen treatment prior to $\mathrm{I} / \mathrm{R}, \mathrm{n}=8)$; and an ebselen control group $(20 \mathrm{mg} / \mathrm{kg}$ ebselen treatment with sham operation, $\mathrm{n}=8$ ). As aforementioned, in the sham group the rats underwent sham operation, and in the I/R group the rats were subjected to $30 \mathrm{~min}$ of ischemia followed by $2 \mathrm{~h}$ of reperfusion. In the ebselen $+\mathrm{I} / \mathrm{R}$ group, the rats received $20 \mathrm{mg} / \mathrm{kg}$ ebselen (MedChemExpress, Monmouth Junction, NJ, USA) intragastrically $24 \mathrm{~h}$ prior to the I/R-inducing surgery and throughout the experimental period. In the ebselen control group, rats received ebselen intragastrically and underwent the sham operation.

Determination of myocardial infarct size. Myocardial infarct size was estimated using triphenyltetrazolium chloride (TTC;
Sigma-Aldrich; Merck KGaA) staining. Heart tissues were perfused with saline to wash out the remaining blood from the coronary vasculature. Then, each heart was sectioned horizontally into $1 \mathrm{~mm}$-thick slices, which were stained with $1 \%$ TTC for $20 \mathrm{~min}$ at $37^{\circ} \mathrm{C}$ and fixed by immersion in $10 \%$ neutral buffered formalin for $6 \mathrm{~h}$ at room temperature. Finally, the infarct area (IA) and area at risk (AAR) were determined using Image J software V1.8.0 (National Institutes of Health, Bethesda, MD, USA). The infarct size was presented as a percentage of the AAR (IA/AAR).

Echocardiography. Echocardiography was performed in rats for assessment of cardiac function prior to and following myocardial I/R injury. Rats were anesthetized with $5.0 \%$ isoflurane and placed supinely on a heating pad, which maintained rat body temperature at $36-37^{\circ} \mathrm{C}$. Anesthesia was maintained by inhalation of $2 \%$ isoflurane, which was driven by $100 \%$ oxygen flow and ventilated using a small animal respirator. Two-dimensional M-mode echocardiograms and pulsed-wave Doppler spectral tracings were obtained using a Vivid 7 echocardiography machine (General Electric Healthcare Corporation, Waukesha, WI, USA) equipped with a $10 \mathrm{MHz}$-phased array transducer. Following this, diastolic left ventricular posterior wall thickness, systolic left ventricular posterior wall thickness, left ventricle diastolic dimension and left ventricle systolic dimension were measured using M-mode tracings. In addition, ejection fraction (EF\%) and percent fractional shortening $(\% \mathrm{FS})$ were calculated.

Histopathological examination of myocardium. Heart tissue samples were obtained and preserved in $2 \%$ glutaraldehyde solution for histopathological examination at the end of myocardial reperfusion. For this, heart tissue specimens were transferred into $70 \%$ ethanol, embedded in paraffin, sectioned into $5 \mu \mathrm{m}$-thick sections, stained with hematoxylin for $5 \mathrm{~min}$ and eosin for $3 \mathrm{~min}$ at room temperature, and examined by light microscopy.

Electron microscopy analysis. Heart tissue samples were obtained at the end of reperfusion and immediately preserved in $2 \%$ glutaraldehyde solution. Samples were fixed in $1 \%$ osmic acid for $2-3 \mathrm{~h}$ at $4^{\circ} \mathrm{C}$, embedded in Epon 812 epoxy resin overnight at $37^{\circ} \mathrm{C}$ and cut into $5 \mu \mathrm{m}$-thick sections. Sections were prepared with acetate double oxygenic uranium for $30 \mathrm{~min}$ and citrate lead stain for $10 \mathrm{~min}$ at room temperature. Following this, the sections were examined using a JEM-1230 transmission electron microscope (BD Biosciences, Tokyo, Japan).

Terminal dUTP nick end-labelling (TUNEL) assay. The TUNEL assay was used to examine myocardial apoptosis. An in situ cell death detection kit (Roche Diagnostics, Indianapolis, IN, USA) was used according to the manufacturer's instructions. The excised heart tissues were fixed for $24 \mathrm{~h}$ using $10 \%$ formic acid solution at room temperature and then cut horizontally into $5 \mu \mathrm{m}$-thick sections. After washing with phosphate-buffered saline (PBS) twice, the tissue sections were preprocessed with $0.1 \%$ Triton X-100 and $0.1 \%$ sodium citrate (freshly prepared) for $15 \mathrm{~min}$. Following this, the sections were incubated for $1 \mathrm{~h}$ at $37^{\circ} \mathrm{C}$ with the commercially prepared labelling mixture and stained with 
4',6-diamidino-2-phenylindole for $5 \mathrm{~min}$ at room temperature. Three slides from each block were evaluated under a fluorescence microscope to determine the percentage of apoptotic cells. In addition, four fields on each slide were examined at the border areas using a defined rectangular field area with a magnification of $\times 20$. The apoptosis rate of cardiomyocytes was presented as the percentage of total cells counted.

Assessment of serum cardiac enzymes. At the end of myocardial reperfusion and prior to sacrifice, $3-5 \mathrm{ml}$ blood samples were collected from the abdominal aorta. Subsequently, the blood samples were centrifuged at 2,000 x $\mathrm{g}$ for $5 \mathrm{~min}$ at room temperature, and the serum obtained was assayed for the activity of cardiac enzymes [creatine kinase (CK), CK-MB isoenzyme and lactate dehydrogenase $(\mathrm{LDH})]$. The activity levels of CK, CK-MB and $\mathrm{LDH}$ were detected using the creatine kinase assay kit (cat. no. A032), creatine kinase MB isoenzyme assay kit (cat. no. E006) and lactate dehydrogenase assay kit (cat. no. A020-1; Nanjing Jiancheng Bioengineering Institute, Nanjing, China), respectively, in accordance with the manufacturer's protocols using an automatic biochemical analyzer.

Western blotting. Heart tissues were lysed in radioimmunoprecipitation assay lysis buffer (EMD Millipore, Billerica, MA, USA) containing complete ${ }^{\mathrm{TM}}$ Protease Inhibitor Cocktail (CWBio, Beijing, China) and homogenized with glass homogenizer until full lysis was achieved. Cell lysates were obtained by centrifuging homogenates at $6,000 \mathrm{xg}$ for $10 \mathrm{~min}$ at $4^{\circ} \mathrm{C}$. Protein concentration was determined using a standard bicinchoninic acid assay. Total protein (10 $\mu \mathrm{g} /$ lane) was separated using SDS-PAGE (10\% gels) and then transferred onto nitrocellulose membranes. The membranes were incubated with anti-cleaved Caspase- 8 (C-Caspase-8; cat. no. 66093-1-Ig), anti-cleaved Caspase-3 (C-Caspase-3; cat. no. 19677-1-AP), anti-B-cell lymphoma 2 (Bcl-2; cat. no. 12789-1-AP), anti-Bcl2-associated X protein (Bax; cat. no. 50599-2-Ig), anti-cleaved poly(ADP-ribose) polymerase (C-PARP; cat. no. 13371-1-AP; all 1:2,000) and anti-GAPDH (cat. no. 60004-1-Ig; 1:3,000; all Wuhan Sanying Biotechnology, Wuhan, China) primary antibodies overnight at $4^{\circ} \mathrm{C}$. Following this, membranes were incubated with horseradish peroxidase-conjugated goat anti-rabbit (cat. no. ab6721) and rabbit anti-mouse (cat. no. ab6728) secondary antibodies (1:5,000; Abcam, Cambridge, MA, USA). Blots were analyzed using an electrochemiluminescence detection system (Bio-Rad Laboratories, Inc., Hercules, CA, USA).

Biochemical assays. To assess the extent of oxidative stress, the activity of GPx and SOD, and levels of malondialdehyde (MDA) and protein carbonyl (PC) in heart tissue were measured. Heart tissue samples were homogenized in PBS, and the supernatant was obtained by centrifuging at $6,000 \mathrm{x}$ g for $10 \mathrm{~min}$ at $4^{\circ} \mathrm{C}$ and preserved at $-80^{\circ} \mathrm{C}$ until analysis. GPX and SOD activity levels were detected using the methods described by Paglia and Valentine (19) and Snow-Lisy et al (20), respectively, and enzyme activity was recorded as $\mathrm{U} / \mathrm{g}$ of protein in heart tissue. MDA levels were measured using a thiobarbituric acid reaction, which was described by Ohkawa et al (21). The assay used spectrophotometric measurements of the color produced during the reaction of thiobarbituric acid with MDA at $535 \mathrm{~nm}$, which were then expressed as nmol/mg of protein. PC levels were measured spectrophotometrically according to the reaction of the carbonyl group with 2, 4-dinitrophenylhydrazine, which is used as a reagent for proteins subjected to metalcatalyzed oxidation as described in detail by Levine et al (22). The result was recorded as nmol carbonyl/mg of protein.

Statistical analysis. Data were expressed as mean \pm standard error of the mean and analyzed using SPSS 18.0 statistical software (SPSS, Inc., Chicago, IL, USA). Statistical analysis was performed using one-way analysis of variance followed by a Student-Newman-Keuls post-hoc test for multi-group comparisons. $\mathrm{P}<0.05$ was considered to indicate a statistically significant difference.

\section{Results}

Ebselen protects against myocardial I/R injury and cardiac dysfunction. To detect whether ebselen protects against myocardial I/R injury, rats were administered ebselen intragastrically prior to the I/R-inducing operation. Rat hearts were subjected to ischemia for $30 \mathrm{~min}$, which was followed by reperfusion for $2 \mathrm{~h}$. Subsequently, hearts were harvested for infarct size evaluation. As depicted in Fig. 1A, the infarct size was significantly increased in the I/R group compared with in the sham group $(\mathrm{P}<0.01)$, which was indicated by the ratio of IA/AAR. Meanwhile, in the ebselen + I/R group, the infarct size was reduced compared with in the $I / R$ group $(P<0.05)$. Additionally, the effect of ebselen on cardiac function was evaluated following myocardial I/R injury via echocardiography. As demonstrated in Fig. $1 \mathrm{~B}$ and $\mathrm{C}$, the $\mathrm{EF} \%$ and \%FS were significantly decreased in the I/R group compared with in the sham group at 7 days post I/R injury induction $(\mathrm{P}<0.05) . \mathrm{EF} \%$ and \%FS were increased in the ebselen $+\mathrm{I} / \mathrm{R}$ group compared with in the $\mathrm{I} / \mathrm{R}$ group $(\mathrm{P}<0.05)$, suggesting ebselen protected against the cardiac dysfunction induced by myocardial I/R injury.

Histopathological and ultrastructural changes of the myocardium were observed using light microscopy (H\&E staining) and electron microscopy. As shown in Fig. 2A, myocardial fibers exhibited an orderly arrangement and no evidence of inflammatory cell infiltration was evident in the sham and ebselen control groups. Meanwhile, focal necrosis of the myocardium, myocardial cell swelling, cell boundary and transverse stripe blur, and neutrophil infiltration in the interstitial area and between cardiomyocytes were observed in the I/R group. In the ebselen $+I / R$ group, these changes were markedly attenuated compared with in the I/R group. However, the myocardial cells still exhibited some swelling and a small amount of inflammatory cell infiltration was observed in the ebselen $+\mathrm{I} / \mathrm{R}$ group. Ultrastructural changes to the organelles in the cytoplasm were observed using electron microscopy. As demonstrated in Fig. 2B, in the sham and ebselen control groups, closely and uniformly arranged myocardial fibers, clear muscle segments, abundant mitochondria and some vacuoles in the cytoplasm were observed. In the I/R group, notable mitochondrial swelling induced by myocardial I/R injury was observed along with abundant fatty and vacuolation degeneration in cardiomyocytes compared with in the sham group. 

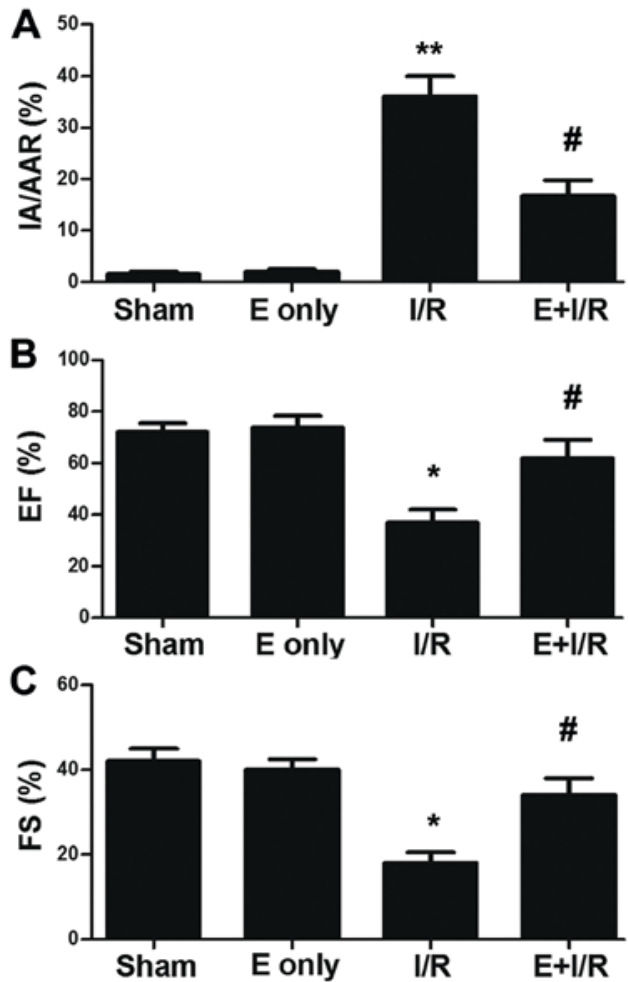

Figure 1. Ebselen attenuates myocardial I/R-induced heart injury. (A) Ebselen reduced myocardial infarct size, which was indicated by the IA/AAR ratio. (B and C) Ebselen attenuated I/R-induced cardiac dysfunction. Cardiac function was determined using echocardiography. Data are expressed as the mean \pm standard error of the mean. ${ }^{* *} \mathrm{P}<0.01$ and ${ }^{*} \mathrm{P}<0.05$ vs. sham group;

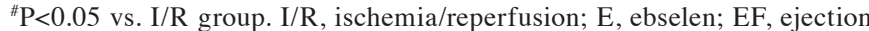
fraction; FS, fractional shortening; IA, infarct area; AAR, area at risk.

The arrangement of myocardial fibers became disordered and damage of myocardial fibrous membrane was also observed. In the ebselen $+\mathrm{I} / \mathrm{R}$ group, these changes were reduced and the cardiac cellular structure remained clearly visible.

Ebselen attenuates I/R-induced myocardial apoptosis and cardiac injury. Narula et al (23) reported that cardiomyocyte apoptosis contributes to myocardial I/R injury. In the current study, it was determined whether ebselen serves an anti-apoptotic role during myocardial I/R injury. TUNEL staining (Fig. 3A) indicated that $\mathrm{I} / \mathrm{R}$ injury significantly induced myocardial apoptosis by $29 \%$ compared with sham treatment $(\mathrm{P}<0.01)$. Furthermore, the rate of myocardial apoptosis in the ebselen $+I / R$ group was reduced compared with in the $\mathrm{I} / \mathrm{R}$ group $(\mathrm{P}<0.05)$. The activity of the cardiac enzymes $\mathrm{CK}$, CK-MB and LDH in serum was also evaluated. As indicated in Fig. 3B-D, the activity levels of serum CK, CK-MB and LDH were markedly increased in the I/R group compared with in the sham group (all $\mathrm{P}<0.05$ ). In turn, ebselen significantly attenuated the activity levels of CK, CK-MB and LDH compared with $\mathrm{I} / \mathrm{R}$ alone (all $\mathrm{P}<0.05$ ).

Ebselen influences the expression of apoptotic proteins. To clarify the mechanisms of ebselen in inhibiting cardiomyocyte apoptosis, the expression of the apoptosis-related proteins C-Caspase-8, C-Caspase-3, Bax, Bcl-2 and C-PARP was assessed. Western blotting (Fig. 4) demonstrated that I/R injury significantly increased the expression of myocardial pro-apoptotic proteins $\mathrm{C}$-Caspase- 8 , Bax (both $\mathrm{P}<0.01$ ), $\mathrm{C}$-Caspase-3 and C-PARP, and decreased the expression of the anti-apoptotic protein Bcl-2 (all $\mathrm{P}<0.05)$ when compared with sham treatment. By contrast, treatment with ebselen suppressed the expression of pro-apoptotic proteins in heart tissues and significantly promoted the expression of $\mathrm{Bcl}-2$ compared with $\mathrm{I} / \mathrm{R}$ alone (all $\mathrm{P}<0.05$ ).

Effects of ebselen on the levels of cardiac antioxidant enzymes, MDA and PC. The effect of ebselen on cardiac antioxidation during $\mathrm{I} / \mathrm{R}$ injury was evaluated. As indicated in Fig. 5A and B, SOD and GPx activity levels were decreased in the $\mathrm{I} / \mathrm{R}$ group compared with in the sham group $(\mathrm{P}<0.01)$. In the ebselen $+\mathrm{I} / \mathrm{R}$ group, the antioxidant enzyme activities were significantly increased compared with in the $\mathrm{I} / \mathrm{R}$ group $(\mathrm{P}<0.05)$. MDA is the final product of lipid peroxidation. MDA levels, as a marker of cardiac oxidative stress, were assayed in heart tissues in the present study. As indicated in Fig. 5C, I/R injury significantly increased the MDA levels in the heart tissues compared with in the sham group $(\mathrm{P}<0.05)$. This I/R-induced increase in MDA levels was significantly prevented by ebselen treatment $(\mathrm{P}<0.05)$. $\mathrm{PC}$ levels in heart tissues were also determined (Fig. 5D). A marked increase in the tissue levels of $\mathrm{PC}$ was observed in the $\mathrm{I} / \mathrm{R}$ group $(\mathrm{P}<0.05$ vs. sham), suggesting increased protein oxidation. Meanwhile, PC levels were significantly decreased in the ebselen $+I / R$ group compared with in the I/R group $(\mathrm{P}<0.05)$.

\section{Discussion}

Coronary reperfusion therapy is an established approach for the management of acute myocardial infarction; however, restoration of blood flow to previously ischemic myocardium results in I/R injury $(24,25)$. Cumulative studies have uncovered that several primary factors, including oxidative stress, intracellular calcium overload, fluctuating physiological $\mathrm{pH}$, the mitochondrial permeability transition pore and inflammation mediate the detrimental effects of myocardial $\mathrm{I} / \mathrm{R}$ injury. These factors collectively induce apoptosis and the death of cardiomyocytes during the I/R process (26-29). Therefore, reducing cardiomyocyte apoptosis may protect the heart against I/R-induced injury.

A number of studies have uncovered that ebselen has beneficial effects in I/R-induced tissue injury, specifically in intestinal (30), lung (31), kidney (32) and sciatic nerve (33) tissues. In the current study, the effects of ebselen on heart injury induced by I/R in vivo were examined. The major findings included the following: i) Ebselen significantly attenuated I/R-induced myocardial infarction and cardiac dysfunction; ii) ebselen attenuated the effects of I/R in reducing SOD and GPx activities and increasing MDA and PC levels in rat hearts, consistent with results reported by Baljinnyam et al (34) in a rabbit model of myocardial I/R injury; iii) ebselen prevented I/R-induced rat heart injury, by attenuating histological and ultrastructural changes, reducing serum CK, CK-MB and LDH activity levels, and decreasing cell apoptosis, which to our knowledge is the first time this has been systematically determined; and iv) ebselen pretreatment markedly decreased the expression of C-Caspase-8, C-Caspase-3, Bax and C-PARP, and increased the expression of $\mathrm{Bcl}-2$. 
A

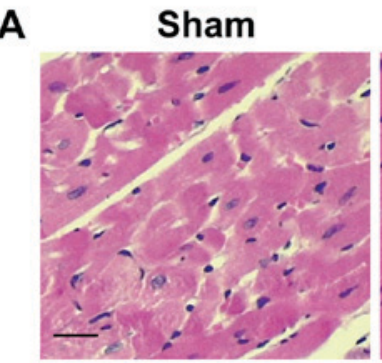

B

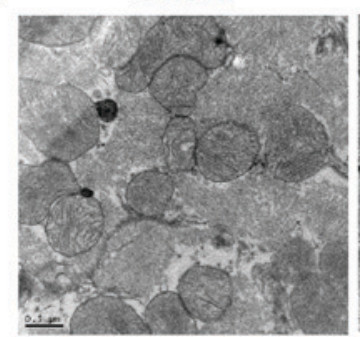

E only

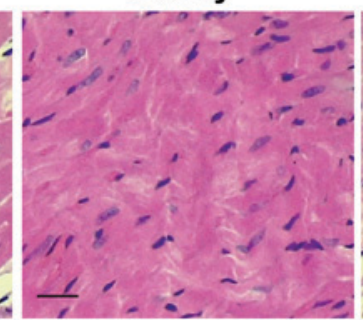

E only

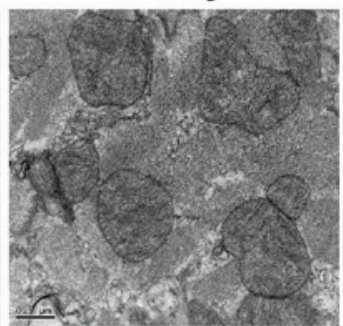

I/R

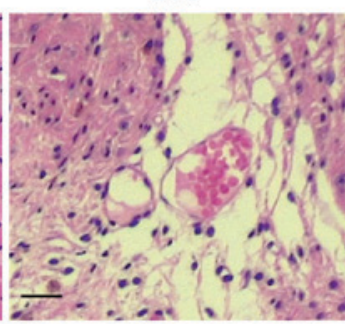

I/R

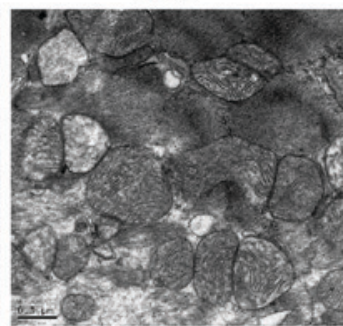

$E+1 / R$

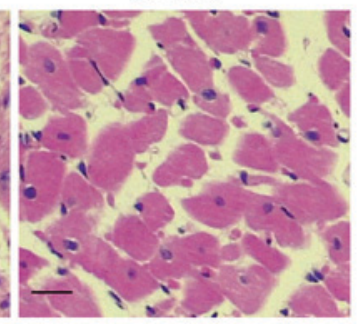

$E+1 / R$

Figure 2. Histopathological and ultrastructural changes of the myocardium following reperfusion. (A) Microscopic histopathological changes of heart tissue determined by hematoxylin and eosin staining. (Sham and E only) Myocardial fibers were arranged in an orderly manner and no inflammatory cell infiltration was evident. (I/R) The arrangement of myocardial fibers was disordered. Cellular edema and breaks or necrosis were evident. In addition, infiltration of inflammatory cells was observed. (E+I/R) Damage was attenuated compared with in the I/R group. Original magnification: $\mathrm{x} 400$. (B) Myocardial ultrastructure changes following reperfusion determined by electron microscopy. (Sham and E only) Myocardial fibers were arranged close together and in an orderly manner. Clear muscle segments and abundant mitochondria were observed. (I/R) Myocardial fibers were disordered in appearance. Severe mitochondrial swelling and abundant fatty and vacuolation degeneration in cardiomyocytes were observed. (E+I/R) Damage was attenuated compared with in the I/R group. Scale bar: $0.5 \mu \mathrm{m}$. I/R, ischemia/reperfusion; E, ebselen.
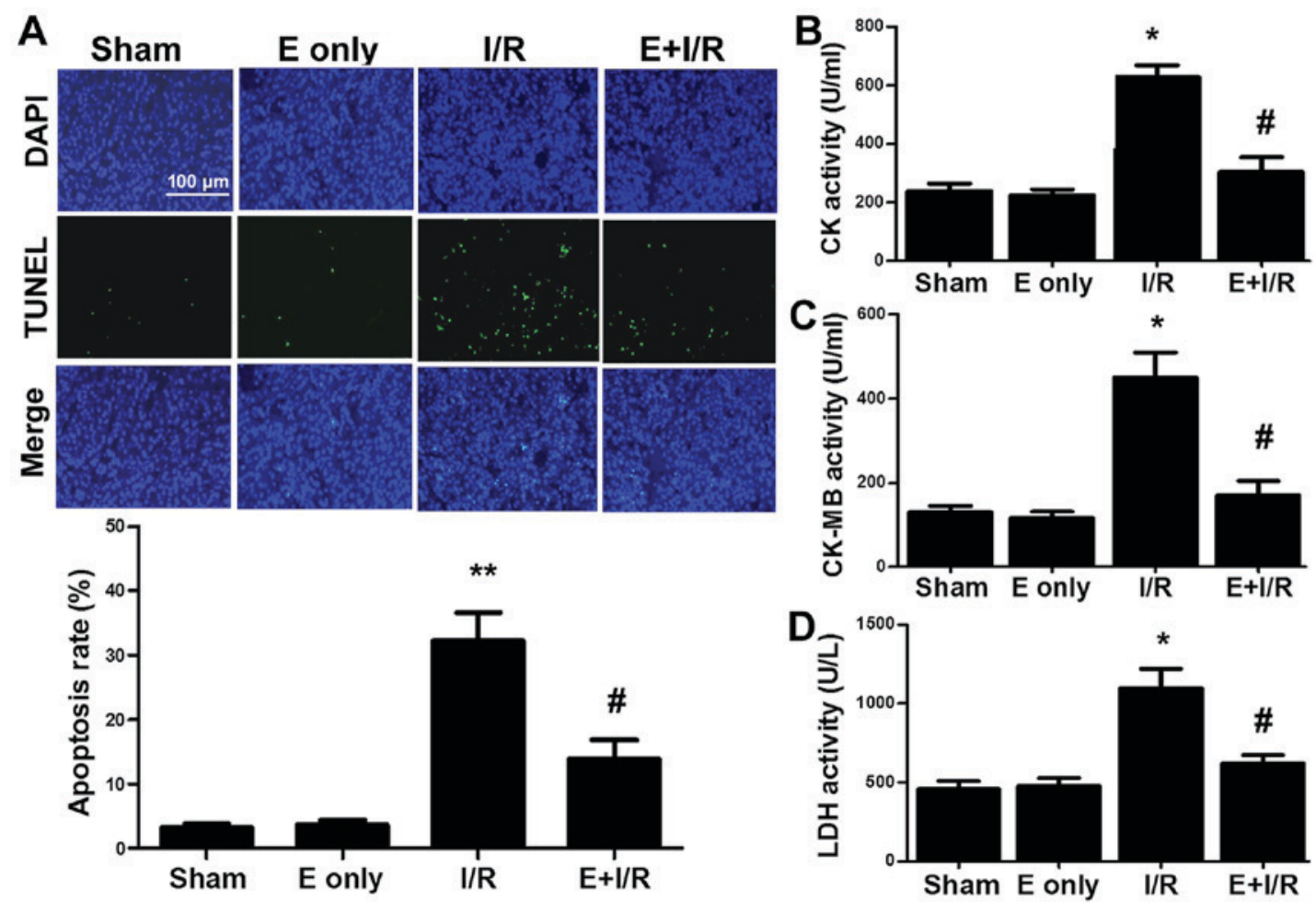

Figure 3. Ebselen attenuates I/R-induced myocardial apoptosis and cardiac injury. (A) Myocardial apoptosis was examined using the TUNEL assay in rat heart sections. Representative images of TUNEL staining (green fluorescence) were captured under a fluorescence microscope. The bar graph indicates the rate of myocardial apoptosis. (B-D) Effects of ebselen on serum cardiac enzymes CK, CK-MB and LDH were demonstrated. Each bar represents the mean of three independent experiments. Data are expressed as the mean \pm standard error of the mean. ${ }^{* *} \mathrm{P}<0.01$ and ${ }^{*} \mathrm{P}<0.05$ vs. sham group; ${ }^{*} \mathrm{P}<0.05$ vs. I/R group. I/R, ischemia/reperfusion; E, ebselen; TUNEL, terminal dUTP nick end-labelling; CK-[MB], creatine kinase [MB isoenzyme]; LDH, lactate dehydrogenase.

In myocardial ischemia, hypoxia and reoxygenation, as principal causes of reperfusion injury, induce an increase in free radical production in cardiac tissues $(35,36)$. ROS and RNS produced through reoxygenation lead to direct oxidative 

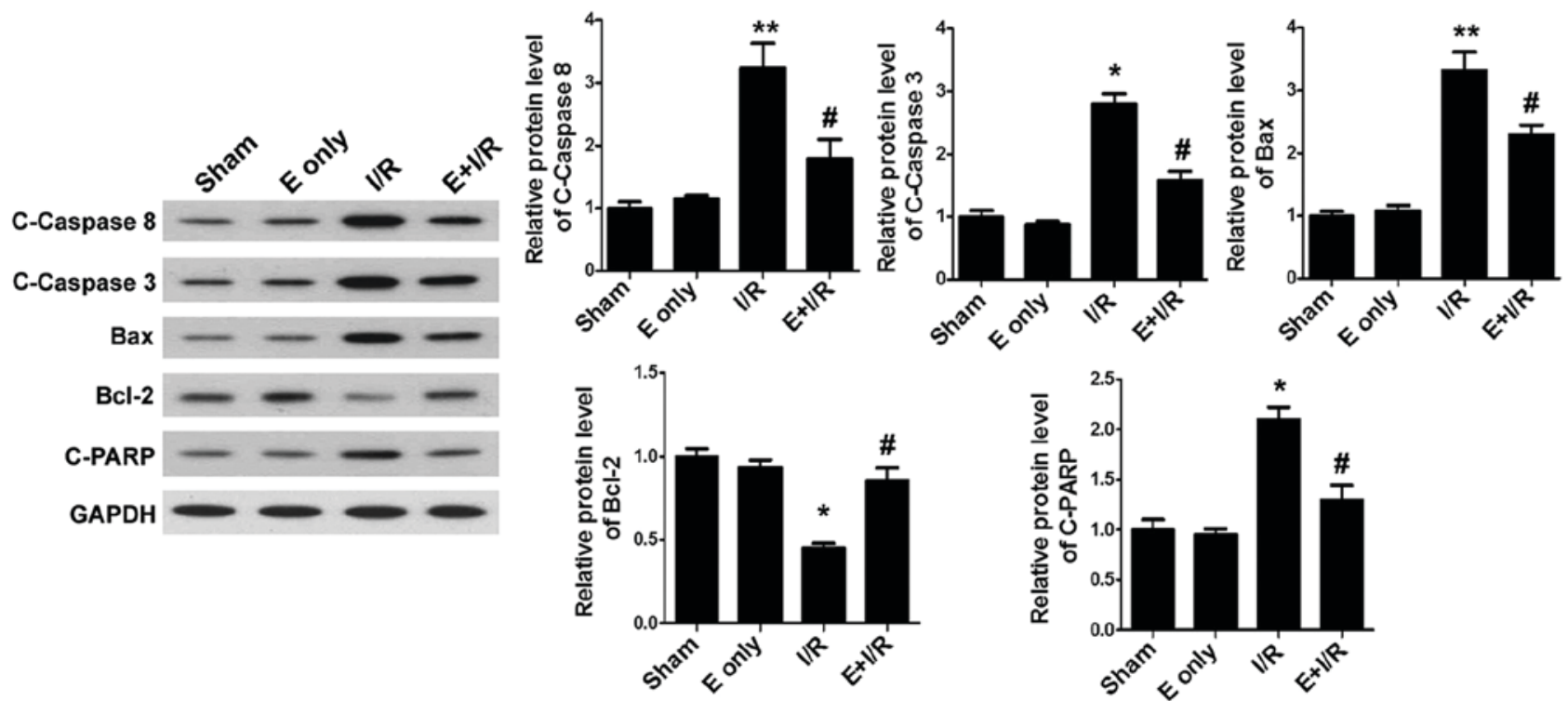

Figure 4. Ebselen influences the expression of apoptosis-related proteins. The effects of ebselen on C-Caspase-8, C-Caspase-3, Bax, Bcl-2 and C-PARP were analyzed by western blotting. The bar graphs indicate the quantification and statistical analysis of western blotting data. Data are expressed as the mean \pm standard error of the mean. ${ }^{*} \mathrm{P}<0.05$ and ${ }^{* *} \mathrm{P}<0.01$ vs. sham group; ${ }^{*} \mathrm{P}<0.05$ vs. I/R group. I/R, ischemia/reperfusion; E, ebselen; C-, cleaved; Bcl-2, B-cell lymphoma 2; Bax, B-cell lymphoma 2-associated X protein; PARP, poly(ADP) ribose.
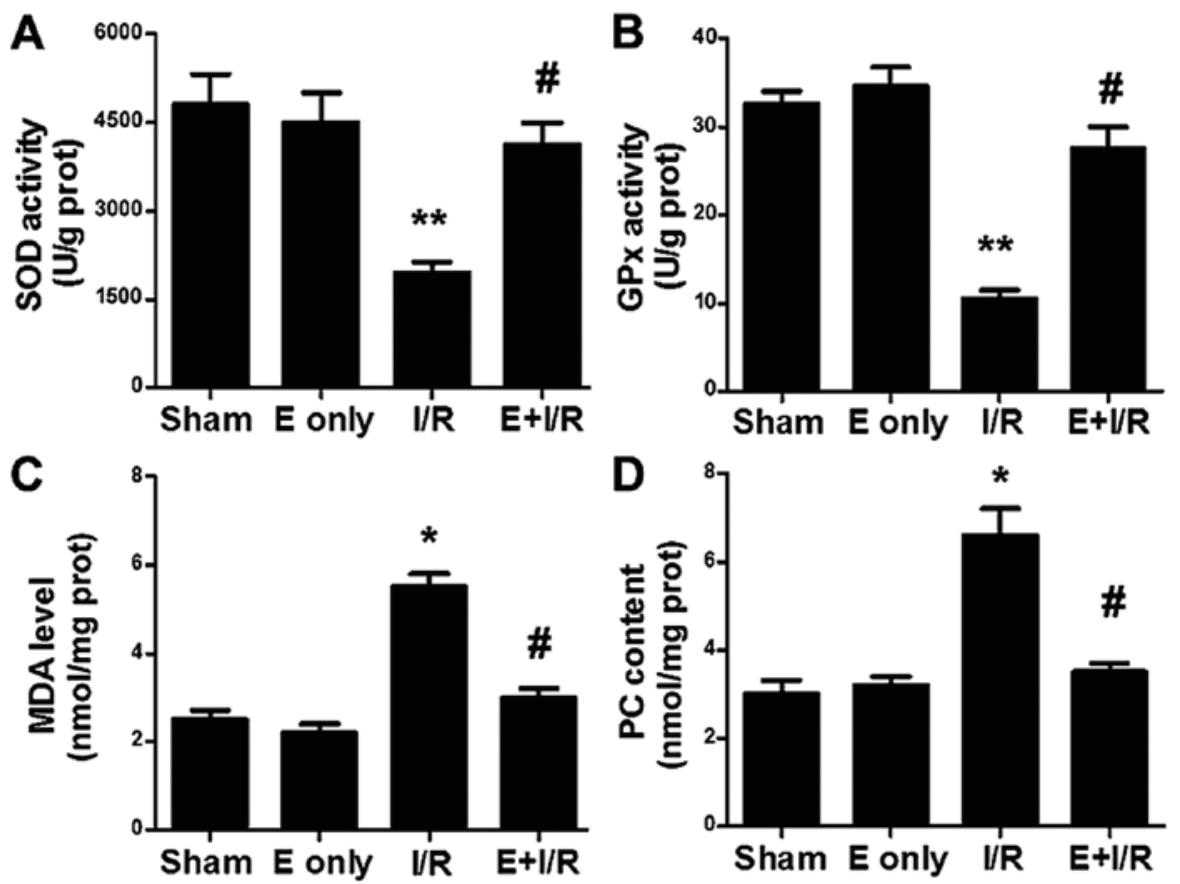

Figure 5. Ebselen influences SOD and GPx activity and MDA and PC levels. Ebselen significantly increased (A) SOD and (B) GPx activity levels in the rat hearts that underwent myocardial I/R injury. Ebselen attenuated the myocardial I/R-induced increase in (C) MDA and (D) PC levels in rat hearts. Data are expressed as the mean \pm standard error of the mean. ${ }^{* *} \mathrm{P}<0.01$ and ${ }^{*} \mathrm{P}<0.05$ vs. sham group; ${ }^{\#} \mathrm{P}<0.05$ vs. I/R group. I/R, ischemia/reperfusion; E, ebselen; prot, protein; SOD, superoxide dismutase; GPx, glutathione peroxidase; MDA, malondialdehyde; PC, protein carbonyl.

damage to cellular components; in particular, damage caused by peroxidation of cellular membrane lipids and oxidation of cellular proteins, which can be measured by assessing MDA and PC levels in the heart tissue, respectively. The present results indicated that I/R injury caused a significant increase in MDA and PC levels in the heart tissues. However, this change was mostly restored in the ebselen-pretreated $I / R$ group. Additionally, it was examined whether ebselen could enhance the antioxidant capacity of rat hearts in the current study. It was identified that myocardial I/R injury decreased SOD and GPx activities, and this effect was attenuated by treatment with ebselen. The effects of ebselen on SOD and GPx activities have been excessively studied in myocardial I/R injury in several animal species including rabbit, in situ swine and neonatal porcine models $(34,37-39)$, and the current results in a rat model are consistent with those of the previous studies. 
However, there appears to be few reports that have determined an increase in MDA and PC levels in myocardial I/R injury, and that ebselen may decrease these levels. Additionally, further investigation is needed to elucidate the role of SOD and GPx in the ebselen-mediated cardioprotection during I/R injury and the anti-oxidant effects of ebselen in myocardial I/R injury, potentially occurring via nitric oxide signaling, in future studies.

A key finding of the present study was that ebselen exhibited various I/R-protective effects via antioxidant and anti-apoptosis functions. Previous studies have indicated that apoptosis induced by oxidative stress serves an important role in the pathogenesis of heart dysfunctions induced by myocardial I/R injury (40-42). Several critical factors, including C-Caspase-8, C-Caspase-3, Bax, Bcl-2 and C-PARP, regulate the process of apoptosis (43-47). In myocardial I/R injury, inhibition of C-Caspase-8, C-Caspase-3 and C-PARP may reduce cardiomyocyte apoptosis $(43,44)$. Furthermore, previous results indicated that overexpression of $\mathrm{Bcl}-2$ and downregulation of Bax inhibited myocyte apoptosis following reperfusion, and protected the heart against I/R injury (45-47). In the current study, the protein expression of genes associated with cell apoptosis was detected using western blotting. According to the results, C-Caspase-8, C-Caspase-3, Bax and $\mathrm{C}$-PARP were overexpressed in the I/R group compared with in the sham group; in turn, the protein expression of these pro-apoptotic factors was decreased in the ebselen-pretreated $\mathrm{I} / \mathrm{R}$ group. By contrast, the expression of $\mathrm{Bcl}-2$ in rat hearts was decreased in the I/R group compared with in the sham group, and subsequently increased by ebselen pretreatment compared with the I/R group. To the best of our knowledge, the present study is the first to indicate that ebselen protects against myocardial I/R injury by upregulating Bcl-2 and downregulating C-Caspase-8, C-Caspase-3, Bax and C-PARP, thus inhibiting I/R-induced apoptosis.

In conclusion, the present study indicated that ebselen could protect rat hearts against I/R injury. Ebselen attenuated I/R-induced cardiomyocyte apoptosis seemingly by increasing the expression of $\mathrm{Bcl}-2$, decreasing the expression of C-Caspase-8, C-caspase-3, Bax and C-PARP, and enhancing the expression and function of antioxidant enzymes. Taken together, the present results support the use of ebselen as an effective therapeutic agent for the treatment of myocardial I/R injury.

\section{Acknowledgements}

The authors would like to thank Dr Jin Gong and Dr Lin Han (Department of Pathology of Renmin Hospital of Wuhan University, Wuhan, China) for technical assistance in histopathological examination of myocardium.

\section{Funding}

The current study was supported by the Scientific Research Project of Yichang City, Hubei in 2013 (grant no. A13301-18).

\section{Availability of data and materials}

Please contact the corresponding author for data requests.

\section{Authors' contributions}

BC and JPZ performed the animal and molecular studies, collected experimental data and drafted the manuscript. GLL, QXR and GL participated in the design of the study and performed the histological examination of the hearts and the statistical analysis. FXW and HJ conceived the study, participated in its design and coordination and helped to draft the manuscript. All authors read and approved the final manuscript.

\section{Ethics approval and consent to participate}

The animal experiments were approved by the Laboratory Animal Ethics Committee of Wuhan University (Wuhan, China).

\section{Patient consent for publication}

Not applicable.

\section{Competing interests}

The authors declare that they have no competing interests.

\section{References}

1. Li C and Jackson RM: Reactive species mechanisms of cellular hypoxia-reoxygenation injury. Am J Physiol Cell Physiol 282: C227-C241, 2002.

2. Mochizuki K, Ohno Y, Kanematsu T, Sakurai-Yamashita Y, Niwa M, Hishikawa Y and Koji T: Possible protection of sinusoidal endothelial cells by endothelin B receptor during hepatic warm ischemia-reperfusion. Surg Today 37: 460-467, 2007.

3. Fröhlich GM, Meier P, White SK, Yellon DM and Hausenloy DJ: Myocardial reperfusion injury: Looking beyond primary PCI. Eur Heart J 34: 1714-1722, 2013.

4. Diez ER, Altamirano LB, García IM, Mazzei L, Prado NJ, Fornes MW, Carrión FD, Zumino AZ, Ferder L and Manucha W: Heart remodeling and ischemia-reperfusion arrhythmias linked to myocardial vitamin d receptors deficiency in obstructive nephropathy are reversed by paricalcitol. J Cardiovasc Pharmacol Ther 20: 211-220, 2015.

5. Elahi MM, Kong YX and Matata BM: Oxidative stress as a mediator of cardiovascular disease. Oxid Med Cell Longev 2: 259-269, 2009.

6. Tahara EB, Navarete FD and Kowaltowski AJ: Tissue-, substrate-, and site-specific characteristics of mitochondrial reactive oxygen species generation. Free Radic Biol Med 46: 1283-1297, 2009.

7. Vassalle C, Pratali L, Boni C, Mercuri A and Ndreu R: An oxidative stress score as a combined measure of the pro-oxidant and anti-oxidant counterparts in patients with coronary artery disease. Clin Biochem 41: 1162-1167, 2008.

8. Rodrigo R, Libuy M, Feliú F and Hasson D: Oxidative stress-related biomarkers in essential hypertension and ischemia-reperfusion myocardial damage. Dis Markers 35: 773-790, 2013.

9. Katsumi H, Nishikawa M, Yasui H, Yamashita F and Hashida M: Prevention of ischemia/reperfusion injury by hepatic targeting of nitric oxide in mice. J Control Release 140: 12-17, 2009.

10. Taki-Eldin A, Zhou L, Xie HY, Chen KJ, Yu D, He Y and Zheng SS: Triiodothyronine attenuates hepatic ischemia/reperfusion injury in a partial hepatectomy model through inhibition of proinflammatory cytokines, transcription factors, and adhesion molecules. J Surg Res 178: 646-656, 2012.

11. Scott MD, Lubin BH, Zuo L and Kuypers FA: Erythrocyte defense against hydrogen peroxide: Preeminent importance of catalase. J Lab Clin Med 118: 7-16, 1991.

12. Sabri A, Hughie HH and Lucchesi PA: Regulation of hypertrophic and apoptotic signaling pathways by reactive oxygen species in cardiac myocytes. Antioxid Redox Signal 5: 731-740, 2003. 
13. Li C, Hu M, Wang Y, Lu H, Deng J and Yan X: Hydrogen sulfide preconditioning protects against myocardial ischemia/reperfusion injury in rats through inhibition of endo/sarcoplasmic reticulum stress. Int J Clin Exp Pathol 8: 7740-7751, 2015.

14. Wang Q, Lin P, Li P, Feng L, Ren Q, Xie X and Xu J: Ghrelin protects the heart against ischemia/reperfusion injury via inhibition of TLR4/NLRP3 inflammasome pathway. Life Sci 186: 50-58, 2017.

15. Hu SY, Zhang Y, Zhu PJ, Zhou H and Chen YD: Liraglutide directly protects cardiomyocytes against reperfusion injury possibly via modulation of intracellular calcium homeostasis. J Geriatr Cardiol 14: 57-66, 2017.

16. Ozaki M, Nakamura M, Teraoka S and Ota K: Ebselen, a novel anti-oxidant compound, protects the rat liver from ischemia-reperfusion injury. Transpl Int 10: 96-102, 1997.

17. Denicola A and Radi R: Peroxynitrite and drug-dependent toxicity. Toxicology 208: 273-288, 2005.

18. National Research Council (US) Institute for Laboratory Animal Research: Guide for the Care and Use of Laboratory Animals. National Academies Press (US), Washington, DC, 1996.

19. Paglia DE and Valentine WN: Studies on the quantitative and qualitative characterization of erythrocyte glutathione peroxidase. J Lab Clin Med 70: 158-169, 1967.

20. Snow-Lisy DC, Sabanegh ES Jr, Samplaski MK, Morris VB and Labhasetwar V: Superoxide dismutase-loaded biodegradable nanoparticles targeted with a follicle-stimulating hormone peptide protect Sertoli cells from oxidative stress. Fertil Steril 101: 560-567, 2014

21. Ohkawa H, Ohishi N and Yagi K: Assay for lipid peroxides in animal tissues by thiobarbituric acid reaction. Anal Biochem 95 351-358, 1979.

22. Levine RL, Garland D, Oliver CN, Amici A, Climent I, Lenz AG Ahn BW, Shaltiel S and Stadtman ER: Determination of carbonyl content in oxidatively modified proteins. Methods Enzymol 186: 464-478, 1990.

23. Narula J, Hajjar RJ and Dec GW: Apoptosis in the failing heart Cardiol Clin 16: 691-710, 1998

24. Piper HM, García-Dorado D and Ovize M: A fresh look at reperfusion injury. Cardiovasc Res 38: 291-300, 1998.

25. Yellon DM and Hausenloy DJ: Myocardial reperfusion injury. N Engl J Med 357: 1121-1135, 2007.

26. Hausenloy DJ and Yellon DM: Myocardial ischemia-reperfusion injury: A neglected therapeutic target. J Clin Invest 123: 92-100, 2013.

27. Zweier JL, Flaherty JT and Weisfeldt ML: Direct measurement of free radical generation following reperfusion of ischemic myocardium. Proc Natl Acad Sci USA 84: 1404-1407, 1987.

28. Heusch G, Boengler K and Schulz R: Inhibition of mitochondrial permeability transition pore opening: The Holy Grail of cardioprotection. Basic Res Cardiol 105: 151-154, 2010.

29. Vinten-Johansen J: Involvement of neutrophils in the pathogenesis of lethal myocardial reperfusion injury. Cardiovasc Res 61 481-497, 2004

30. Guven A, Tunc T, Topal T, Kul M, Korkmaz A, Gundogdu G, Onguru $\mathrm{O}$ and Ozturk H: Alpha-lipoic acid and ebselen prevent ischemia/reperfusion injury in the rat intestine. Surg Today 38 : 1029-1035, 2008.

31. Hamacher J, Stammberger U, Weber E, Lucas R and Wendel A: Ebselen improves ischemia-reperfusion injury after rat lung transplantation. Lung 187: 98-103, 2009.
32. Kizilgun M, Poyrazoglu Y, Oztas Y, Yaman H, Cakir E, Cayci T, Akgul OE, Kurt YG, Yaren H, Kunak ZI, et al: Beneficial effects of $\mathrm{N}$-acetylcysteine and ebselen on renal ischemia/reperfusion injury. Ren Fail 33: 512-517, 2011.

33. Ozyigit F, Kucuk A, Akcer S, Tosun M, Kocak FE, Kocak C, Kocak A, Metineren H and Genc O: Different dose-dependent effects of ebselen in sciatic nerve ischemia-reperfusion injury in rats. Bosn J Basic Med Sci 15: 36-43, 2015.

34. Baljinnyam E, Hasebe N, Morihira M, Sumitomo K, Matsusaka T, Fujino T, Fukuzawa J, Ushikubi $F$ and Kikuchi K: Oral pretreatment with ebselen enhances heat shock protein 72 expression and reduces myocardial infarct size. Hypertens Res 29: 905-913, 2006.

35. Hori $\mathrm{M}$ and Nishida $\mathrm{K}$ : Oxidative stress and left ventricular remodelling after myocardial infarction. Cardiovase Res 81: 457-464, 2009

36. Zhou S, Sun W, Zhang Z and Zheng Y: The role of Nrf2-mediated pathway in cardiac remodeling and heart failure. Oxid Med Cell Longev 2014: 260429, 2014.

37. Maulik N and Yoshida T: Oxidative stress developed during open heart surgery induces apoptosis: Reduction of apoptotic cell death by ebselen, a glutathione peroxidase mimic. J Cardiovasc Pharmacol 36: 601-608, 2000.

38. Chen Y, Liu J, Li S, Yan F, Xue Q, Wang H, Sun P and Long C: Histidine-tryptophan-ketoglutarate solution with added ebselen augments myocardial protection in neonatal porcine hearts undergoing ischemia/reperfusion. Artif Organs 39: 126-133, 2015.

39. Babiker FA, Al-Jarallah A and Joseph S: Understanding pacing postconditioning-mediated cardiac protection: A role of oxidative stress and a synergistic effect of adenosine. J Physiol Biochem 73: 175-185, 2017.

40. Zhao ZQ: Oxidative stress-elicited myocardial apoptosis during reperfusion. Curr Opin Pharmacol 4: 159-165, 2004.

41. Pu J, Yuan A, Shan P, Gao E, Wang X, Wang Y, Lau WB, Koch W, Ma XL and He B: Cardiomyocyte-expressed farnesoid-X-receptor is a novel apoptosis mediator and contributes to myocardial ischaemia/reperfusion injury. Eur Heart J 34: 1834-1845, 2013.

42. Zhu Z, Zhu J, Zhao X, Yang K, Lu L, Zhang F, Shen W and Zhang R: All-trans retinoic acid ameliorates myocardial ischemia/reperfusion injury by reducing cardiomyocyte apoptosis. PLoS One 10: e0133414, 2015.

43. Xie H, Zhang J, Zhu J, Liu LX, Rebecchi M, Hu SM and Wang C: Sevoflurane post-conditioning protects isolated rat hearts against ischemia-reperfusion injury via activation of the ERK1/2 pathway. Acta Pharmacol Sin 35: 1504-1513, 2014

44. Szobi A, Rajtik T, Carnicka S, Ravingerova T and Adameova A Mitigation of postischemic cardiac contractile dysfunction by CaMKII inhibition: Effects on programmed necrotic and apoptotic cell death. Mol Cell Biochem 388: 269-276, 2014.

45. Ma N, Bai J, Zhang W, Luo H, Zhang X, Liu D and Qiao C: Trimetazidine protects against cardiac ischemia/reperfusion injury via effects on cardiac miRNA-21 expression, Akt and the Bcl-2/Bax pathway. Mol Med Rep 14: 4216-4222, 2016.

46. Cui X, He Z, Liang Z, Chen Z, Wang H and Zhang J: Exosomes from adipose-derived mesenchymal stem cells protect the myocardium against ischemia/reperfusion injury through Wnt/ $\beta$-catenin signaling pathway. J Cardiovasc Pharmacol 70: 225-231, 2017.

47. Hu L, Cai N and Jia H: Pterostilbene attenuates myocardial ischemia-reperfusion injury via the phosphatidylinositol 3'-kinase-protein kinase B signaling pathway. Exp Ther Med 14: 5509-5514, 2017 